\title{
Artificial Intelligence-Based Computed Tomography Imaging Characteristics for the Diagnosis Effect of High Flow Nasal Cannula in the Treatment of Patients with Advanced Lung Cancer and Chronic Obstructive Pulmonary Disease
}

\author{
Xiaobin Tang $\mathbb{D}^{1}$ and Jiang Yuan $\mathbb{D}^{2}$ \\ ${ }^{1}$ Department of Intensive Care Unit, Chenzhou First People's Hospital (The First Affiliated Hospital of Xiangnan College), \\ Chenzhou 423000, Hunan, China \\ ${ }^{2}$ Department of Dermatology, Chenzhou First People's Hospital (The First Affiliated Hospital of Xiangnan College), \\ Chenzhou 423000, Hunan, China \\ Correspondence should be addressed to Jiang Yuan; 1811031054@nbu.edu.cn
}

Received 9 July 2021; Revised 12 August 2021; Accepted 14 August 2021; Published 6 October 2021

Academic Editor: Gustavo Ramirez

Copyright (c) 2021 Xiaobin Tang and Jiang Yuan. This is an open access article distributed under the Creative Commons Attribution License, which permits unrestricted use, distribution, and reproduction in any medium, provided the original work is properly cited.

Objective. This study was to compare the effects of symptomatic treatment and high flow nasal cannula (HFNC) treatment on patients with advanced lung cancer complicated with chronic obstructive pulmonary disease (COPD) and to explore the clinical application effect of HFNC treatment in such patients. Methods. 80 patients with advanced lung cancer and COPD admitted to the hospital were selected as the research objects. They were randomly divided into a control group $(n=40)$ and an observation group $(n=40)$. The computed tomography (CT) image data of all patients were classified. The neural network was trained to obtain the network weights. Based on surgery, radiotherapy, and chemotherapy, patients in the control group received anti-inflammatory, phlegm, and other symptomatic treatments, while patients in the observation group received HFNC treatment on this basis. The blood gas analysis results, clinical symptoms (cough, wheezing, rales, etc.), inflammatory factors (high-sensitivity C-reactive protein (hs-CRP), plateletcrit (PCT), tumor necrosis factor- $\alpha$ (TNF- $\alpha$ ), and interleukin-6 (IL-6)), and quality of life of the two groups were compared and analyzed. Results. When the model MSE was the smallest, the corresponding hidden layer neuron node value was 49 , so 49 was set as the optimal number of hidden layer neuron nodes. CT images were imported into the constructed model system, and the model diagnosis system could still diagnose and classify under the premise that the pathological characteristics were not obvious. There was no significant difference in clinical data between the two groups of patients before treatment $(P>0.05)$. After treatment, the clinical symptoms, arterial partial pressure of carbon dioxide $\left(\mathrm{PaCO}_{2}\right)$, arterial partial pressure of oxygen $\left(\mathrm{PaO}_{2}\right)$, hs-CRP, PCT, TNF- $\alpha$, and IL- 6 levels were greatly reduced; those of patients in the observation group were much better in contrast to those of the control group $(P<0.05)$. The total effective rate in the observation group was $97.5 \%$, and the effective rate in the control group was $87.5 \%$. After treatment, the functional assessment of cancer therapy-lung (FACT-L) score was obviously higher than that before treatment $(P<0.05)$. After treatment, the quality of life in the observation group was increased by $45.69 \%$ compared with that before treatment, and the quality of life in the control group was increased by $35.77 \%$. Conclusion. HFNC therapy can improve the lung function of patients with advanced lung cancer and COPD, alleviate the development of the disease, and improve the quality of life of patients.

\section{Introduction}

Chronic obstructive pulmonary disease (COPD) is a common clinical chronic multiple respiratory diseases, and the main feature is irreversible airflow limitation [1]. In recent years, the incidence rate has been increasing year by year, and the disability rate and fatality rate are as high as $50 \%$. The long-term development of the disease will seriously affect lung function, induce respiratory failure, and bring serious harm to human health $[2,3]$. The global incidence rate of 
people over 40 years old has reached $9 \%-10 \%$, and the characteristics of COPD etiology in China are also particularly prominent. The population of chronic obstructive deaths accounts for about $1 / 3$ of the total deaths in the world [4]. The commonly used clinical methods include anti-infection, relieving cough and reducing phlegm, correcting electrolyte disturbances, and other basic treatment methods. In the treatment of pediatric patients, the diagnosis and treatment method is high flow nasal cannula (HFNC) therapy [5], and positive pressure ventilation therapy and mask oxygen are also used. However, this method requires the patient to have a higher tolerance. During the process, it is easy to leak air, the patient's comfort is poor, and the effect is not ideal [6]. HFNC is widely used in foreign countries and relatively few in China. It is a new type of noninvasive respiratory support method, and its tolerance and comfort are very good [7]. The researchers of Yuste et al. [8] compared the clinical effects of HFNC and continuous noninvasive ventilator-assisted breathing in the treatment of COPD with respiratory failure and found that the respiratory rate, heart rhythm, and total clinical efficiency of patients with HFNC had significant effects, and the adverse reactions were low. In addition, HFNC was found to be easier to operate and care for.

COPD is an independent risk factor for lung cancer. Lung cancer originates from malignant tumors of the bronchial mucosa or glands, and both the mortality and incidence rate occupy the first place in tumors [9]. COPD can increase the risk of cancer through some potential mechanisms such as chronic inflammation, ciliary motor dysfunction, gene mutation, and oxidation/antioxidant imbalance [10]. $20 \%-40 \%$ of lung cancer patients undergoing surgical treatment have COPD. COPD increases the surgical risk of lung cancer patients, reduces the quality of patients, and has negative effects on postoperative complications and postoperative generation [11]. The application of computed tomography (CT) under artificial intelligence in medicine has greatly improved the lung imaging quality of traditional technology and improved the detection and identification capabilities of CT images in lung diseases. The definition of imaging information has been improved, and the image information has become more complete. The characteristic display and detection rate of diseases on the image have been improved [12]. The use of artificial intelligence deep learning can automatically mark information such as the size and nature of the nodules and effectively shorten the diagnosis and treatment time. Traditional scans sometimes cause missed scans due to improper control of the patient's breathing movement, but the artificial intelligence CT can effectively overcome this shortcoming and solve the missed diagnosis of heartbeat and respiratory movement problems [13]. Backpropagation (BP) is also a feedforward neural network. It is a common algorithm in the field of fault diagnosis. It is also an artificial intelligence learning algorithm that has been applied in various fields in recent years [14]. It is the reverse algorithm of the forward algorithm. It has good application effects in CT image processing, forecasting, and prediction. Due to the good nonlinear mapping ability of BPNN, its application in image recognition, information processing, model identification, etc. is also quite good, and the calculations when training deep models have become easier and easier [10]. The introduction of BP neural network in CT image processing can provide a gray-level cooccurrence matrix for CT images, which can effectively segment the image, and has strong selflearning and adaptability.

Studies have confirmed that HFNC treatment for COPD has significant clinical effects, and there is also evidence that HFNC treatment is an important measure to improve respiratory function during the perioperative period of lung cancer, radiotherapy, and chemotherapy. However, there is no report on whether HFNC treatment can be applied to patients with advanced lung cancer and COPD. In this study, the BPNN was applied to the analysis and diagnosis of patients' CT images, effectively reducing the rate of misdiagnosis. The HFNC treatment for patients with advanced lung cancer and COPD would not only help improve the respiratory function of such patients but also help relieve the body's inflammatory response and promote the quality of life of patients, so as to provide a reference for future diagnosis and treatment of lung cancer.

\section{Methods and Materials}

\subsection{Selection and Grouping of Patients}

2.1.1. Subject Selection. 80 patients with advanced lung cancer and COPD were selected as the research objects. In the control group $(n=40)$, there were 15 males and $25 \mathrm{fe}$ males, with an average age of $59.67 \pm 6.23$ years old, which was taken as the experimental group. In the observation group $(n=40)$, there were 18 males and 22 females, with an average age of $57.98 \pm 5.89$ years old. After a comparative analysis of the general data between the two groups, it was found that the difference was not statistically significant $(P<0.05)$. This experiment was reviewed and approved by the Medical Ethics Committee, and relevant personnel signed the informed consent forms.

The inclusion criteria were defined as follows: patients with mid-advanced lung cancer diagnosed by CT and histology (the clinical staging was based on the eighth edition of the tumor node metastasis (TNM) staging system promulgated and implemented by the International Association for the Study of Lung Cancer (IASLC) in January 2017; patients with COPD conforming to the diagnostic criteria given in Chronic Obstructive Pulmonary Disease Diagnosis and Treatment Guidelines; patients who could cooperate with medical workers independently; those who had not interrupted the treatment in the hospital; those who met the treatment indications; and those with complete clinical data. The exclusion criteria were given as follows: those with organic disease, hematopoietic system, and other serious diseases; those with mental illness; those with poor treatment compliance; those with communication disorders; those combined with respiratory diseases such as bronchial asthma, bronchiectasis, and tuberculosis; patients who discontinued treatment due to multiple reasons; and patients with tumors or metastatic tumors in other parts. 
2.1.2. Treatment Method. According to the random number table, the patients were divided into 2 groups, with 40 cases in each group; and they were set as the control group and the observation group, respectively. On the basis of surgery, radiotherapy, and chemotherapy, patients in the control group received symptomatic treatment such as anti-inflammatory and phlegm-reducing treatments, and patients in the observation group received HFNC treatment on this basis. Among them, patients with surgical indications were treated with lung cancer surgery, and the radiotherapy and chemotherapy regimens were selected according to relevant guidelines and combined with the patient's own situation. The basic treatment specifications were formulated in accordance with the "Guidelines for the Diagnosis and Treatment of Chronic Obstructive Pulmonary Disease" (2017 version). The symptomatic treatment plan included the regular comprehensive treatment: controlling infection, relieving airway spasms, relieving cough and expectorant, correcting water-electrolyte and acid-base imbalance, shortterm use of glucocorticoids, and nutritional support treatment.

The HFNC treatment plan includes reasonably controlling the oxygen concentration and maintaining it at about $30 \%$, maintaining the oxygen flow rate at $2-10 \mathrm{~L} / \mathrm{min}$, controlling the gas temperature in the humidifier at $37^{\circ} \mathrm{C}$, adjusting the treatment parameters timely according to the actual condition to meet the treatment needs of patients, and lasting the treatment for 14 days. It had to pay attention to choosing the right type of nasal match, adjust the tightness of the nasal match-fixing belt, closely observe the changes in the condition, and adjust the oxygen flow in time to avoid the occurrence of undesirable complications.

Inflammatory factors were detected with the following steps. $4 \mathrm{~mL}$ of peripheral blood was taken from the patient on an empty stomach in the morning, dried in a test tube, centrifuged at $3000 \mathrm{r} / \mathrm{min}$ for 10 minutes to collect the supernatant, which was reserved at $-20^{\circ} \mathrm{C}$. The BIO-RAD Model 550 microplate reader from Toshiba Electric Co. was used to detect the interleukin 8 (IL-8), interleukin 6 (IL-6), high-sensitivity C-reactive protein (hs-CRP), and plateletcrit (PCT). The detection steps were strictly in accordance with the instructions of the kit. The model of the enzyme-linked immunosorbent assay kit was $48 \mathrm{~T} / 96 \mathrm{~T}$.

2.2. Observation Indicators. The blood gas analysis result, clinical symptoms, inflammatory factors (hs-CRP, PCT, TNF- $\alpha$, and IL-6), and quality of life of patients were compared and analyzed between the two groups. The blood gas analysis results mainly included arterial partial pressure of carbon dioxide $\left(\mathrm{PaCO}_{2}\right)$ and arterial partial pressure of oxygen $\left(\mathrm{PaO}_{2}\right)$. The clinical symptoms included coughing, wheezing, and rales. The quality of life was evaluated using the functional assessment of cancer therapy-lung (FACT-L) (4.0) Chinese version, which was one of the Functional Assessments of Cancer Therapy (FACT). The system was developed by Cella et al. of Rush-Presbyterian-St. Luke Medical Center in Chicago, USA. It consists of a general scale (common module) FACT-G that measures the common part of the quality of life of cancer patients and some specific cancer subquantities. FACT-G consists of 34 items, divided into five categories: physical status (8 items), social/family status (8 items), relationship with doctors (3 items), emotional status ( 7 items), and functional status (8 items). The last item of each part is a total evaluation of the part by the patient (used as a total evaluation and weighted scoring), and these items are not included in the calculation of the scores of each part. The scale for specific cancers was composed of common modules plus their own specific modules (the specific module also had a general evaluation item for this part, which was not included when scoring). The details are given in Table 1.

2.3. Image Processing Using BP Algorithm. The CT images of patients diagnosed with lung cancer and COPD in our hospital were selected as the research objects. The obtained CT images were delivered to the workstation, and Functool II software was used to process the images. Postprocessing workstation was Vitrea 3.9 version. Firstly, the image was preprocessed: the image size of $256 * 256$, color processing, and feature extraction. Secondly, the image was divided into a verification set and a training set according to a ratio of 1 : 3. The training set was adopted to train the BPNN, and the specific process is shown in Figure 1. Finally, the verification set was used to verify the diagnosis of lung cancer by the BPNN. The flowchart of the CT-assisted diagnosis system is shown in Figure 2.

2.4. Topology of BPNN. BP is a multilayer positive feedback neural network trained according to the error backpropagation algorithm.

In the training process, the BPNN uses a form of supervised learning, which uses a gradient descent program to train step by step and refresh the weighting coefficients and thresholds. The detailed training steps were as follows:

Step 1: initialization: to know the topology of neural network clearly

Step 2: input: to read the input sequence

Step 3: calculation: the calculated values $H$ and $Y$ of the hidden layer and the output layer were given as follows:

$$
\left\{\begin{array}{l}
H_{j}=f\left(\sum_{i=1}^{n} \omega_{i j}-\theta_{j}\right), \\
Y_{k}=\sum_{j=1}^{l} H_{J} \omega_{j k}-\theta_{k} .
\end{array}\right.
$$

In the above equation, $\theta_{j}$ was the corresponding threshold of the hidden layer, and $\theta_{k}$ referred to the corresponding threshold of the output layer.

Step 4: calculation: the mean square error (MSE) between the output value $Y$ and the expected output value $Y$ was expressed as follows:

$$
E_{\mathrm{MS}}\left(Y_{K}\right)=E\left(Y_{k}-Y_{k}\right)^{2} \text {. }
$$


TABLE 1: The fields of FAC-L and their scoring (rough scoring) method.

\begin{tabular}{lccc}
\hline Item & Score & Score range & Sum of scores \\
\hline Physical condition & 7 & $0 \sim 28$ & $1+2+3+4+5+6+7$ \\
Social/family status & 7 & $0 \sim 28$ & $9+10+11+12+13+14+15$ \\
Relationship with the doctor & 2 & $0 \sim 8$ & $17+18$ \\
Emotional state & 6 & $0 \sim 24$ & $20+21+22+23+24+25$ \\
Functional status & 7 & $0 \sim 28$ & $27+28+29+30+31+32+33$ \\
Lung cancer specific module & 9 & $0 \sim 36$ & $35+36+37+38+39+40+41+42+43$ \\
Total score & 38 & $0 \sim 152$ & $1+2+\ldots+42+43$ \\
\hline
\end{tabular}

Note. Items $1 \sim 7,9,13,20,22 \sim 24,35 \sim 36,38 \sim 39,41$, and 43 were reverse items, and forward transformation should be performed when calculating item scores.

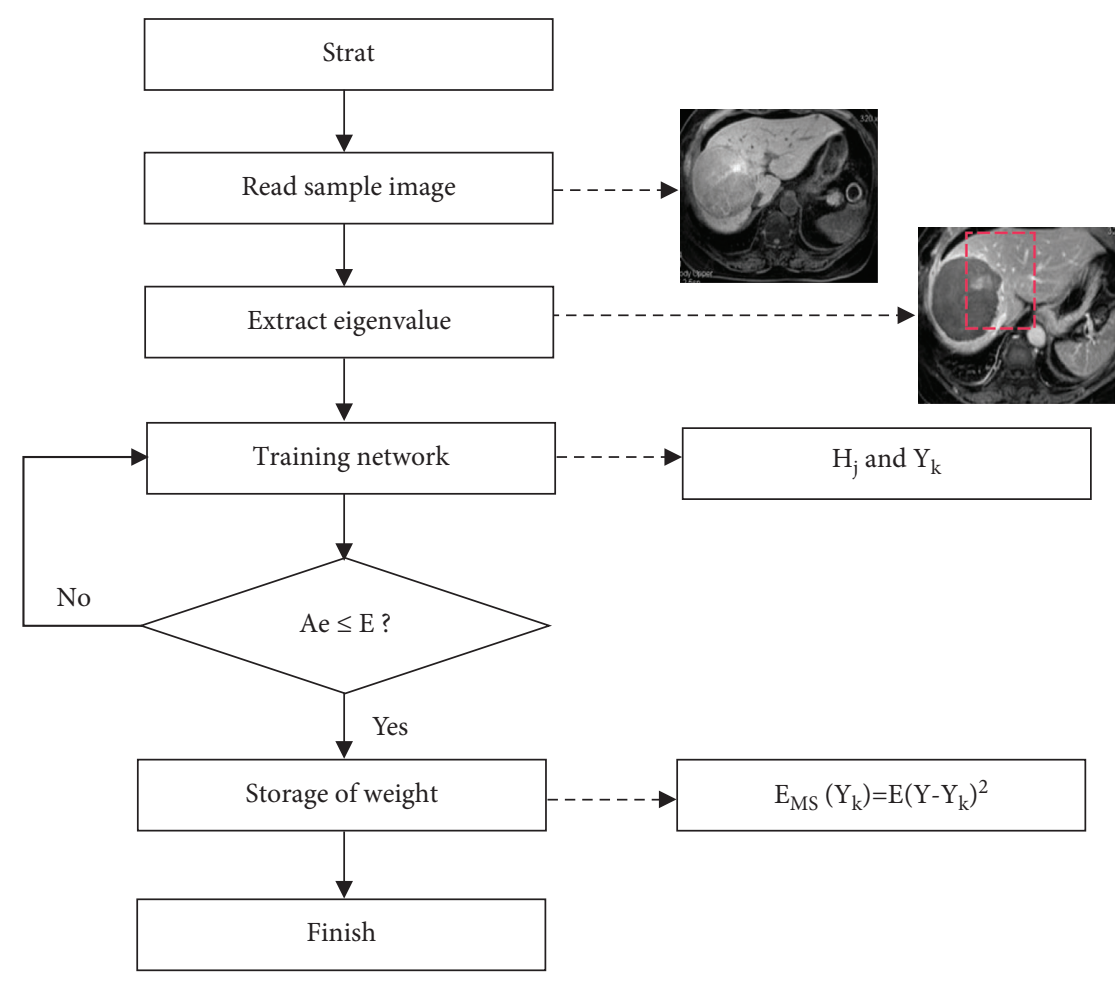

FIGURE 1: Training flowchart of BPNN.

Step 5: adjustment: the weighting coefficients of the hidden layer and the output layer were as follows:

$$
\left\{\begin{array}{l}
\omega_{i j}=\omega_{i j}+\eta H_{j}\left(1-H_{j}\right) x_{i} \sum_{k=1}^{m} \omega_{j k} E_{\mathrm{MS}}\left(Y_{k}\right), \\
\omega_{j k}=\omega_{j k}+\eta H_{j} E_{\mathrm{MS}}\left(Y_{k}\right) .
\end{array}\right.
$$

In the above equation, $\eta$ was the learning efficiency. $\omega$ was the value of each parameter of the matrix.

Step 6: return to Step 3 until the error requirement can be satisfied.

In this study, a BPNN structure with a single hidden layer was adopted.

The characteristics of the training data in the BPNN determined the number of neural nodes in the input layer and output layer. The value range was determined by using the optimized empirical equation. After the experiment, the value corresponding to the minimum error obtained after training was undertaken as the number of hidden nodes. The empirical equation was given as follows:

$$
l=\frac{n \cdot \sqrt{m \cdot n}}{2} .
$$

The sigmoid function was the activation function, and the parameters can be adjusted to achieve precise control of the model. Its expression was written as follows:

$$
S(x)=\frac{1}{1+e^{-x}} \text {. }
$$

According to the principle of the smallest weighting coefficient, the initial value and threshold of the training sample were set.

The empirical equation for calculating the number of hidden layer nodes was expressed as follows: 


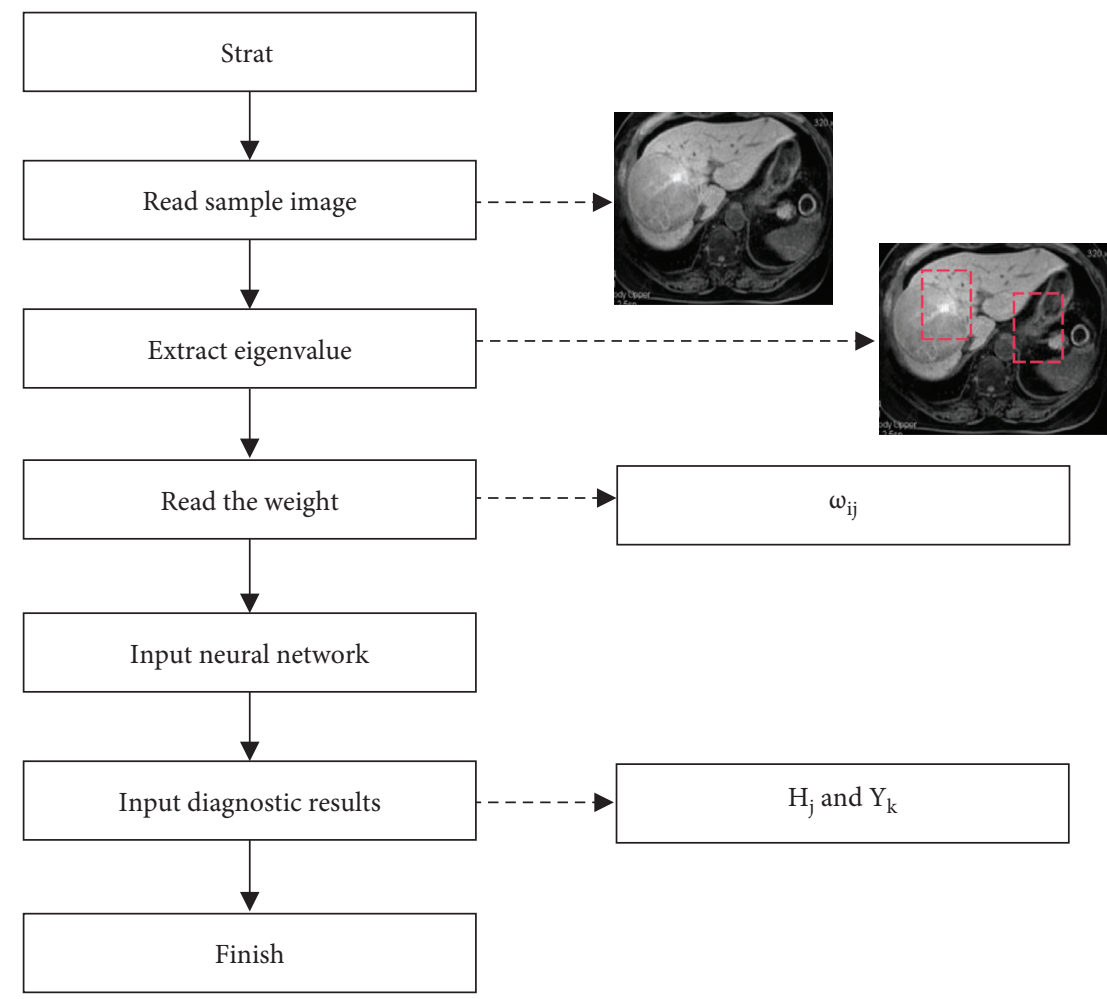

FIGURE 2: Diagnostic flowchart of CT-assisted diagnosis system.

$$
\begin{aligned}
& P=n-1, \\
& p=\sqrt{n+m+a} .
\end{aligned}
$$

2.5. Statistical Analysis. The data was processed using SPSS 20.0 for statistical analysis. The clinical symptoms and the incidence of adverse reactions were all count data. The comparison between groups was performed by chi-square test. The $\mathrm{PaCO}_{2}, \mathrm{PaO}_{2}$, hs-CRP, PCT, TNF- $\alpha$, IL-6, and FAC-L scores were all normally distributed measurement data, expressed as mean \pm standard deviation. The independent sample $t$-test was used for comparison between two groups, paired $t$-test was used for comparison before and after the intervention, and $P<0.05$ was considered statistically significant.

\section{Results and Analysis}

3.1. MSE Curve of Neural Network. As shown in Figure 3, when the model MSE was the smallest in the optimal interval, the corresponding hidden layer neuron node value was 49 , so 49 was set as the optimal number of hidden layer neuron nodes.

3.2. CT Images. The CT symptoms of patients with advanced lung cancer were more obvious. Figures 4(a) and 4(b) show CT images of two patients. The patient's symptoms include shortness of breath, pleural effusion, and different degrees of shortness of breath in the late stage of lung cancer that had regional spread. The normal tissue fluid produced by the lung and myocardium was returned from the lymph nodes in the middle of the chest. Figures 4(c) and 4(d) show the CT image before the algorithm reconstruction. Compared with the $\mathrm{A}$ and $\mathrm{B}$ images, the graininess was more obvious. It showed that the CT image was clearer after image reconstruction.

3.3. Comparison of Inflammatory Factor Test Results. Figure 5 reveals that the content of inflammatory factors (hsCRP, PCT, TNF- $\alpha$, and IL- 6 ) in the two groups before and after treatment showed the same change trend.

A was the IL- 6 comparison between the two groups, B was the TNF- $\alpha$ comparison between the two groups, $C$ was the PCT comparison between the two groups, and D was the hs-CRP comparison between the two groups. Before treatment, there was no obvious difference in the contents of inflammatory factors between the two groups $(P>0.05)$. The contents of inflammatory factors in the latter two groups were reduced, but those in the observation group were significantly lower in contrast to those in the control group $(P<0.05)$.

3.4. Comparison of $\mathrm{PaCO}_{2}$ and $\mathrm{PaO}_{2}$ before and after Treatment. As illustrated in Figure 6, the $\mathrm{PaO}_{2}$ and $\mathrm{PaCO}_{2}$ levels of the two groups of patients before treatment were not significantly different $(P>0.05)$. A was the $\mathrm{PaO}_{2}$ comparison between the two groups, and $\mathrm{B}$ was the $\mathrm{PaCO}_{2}$ comparison between the two groups. After treatment, the $\mathrm{PaO}_{2}$ content of the observation group was much higher than that of the control group $(P<0.05)$, and the $\mathrm{PaCO}_{2}$ content was much 


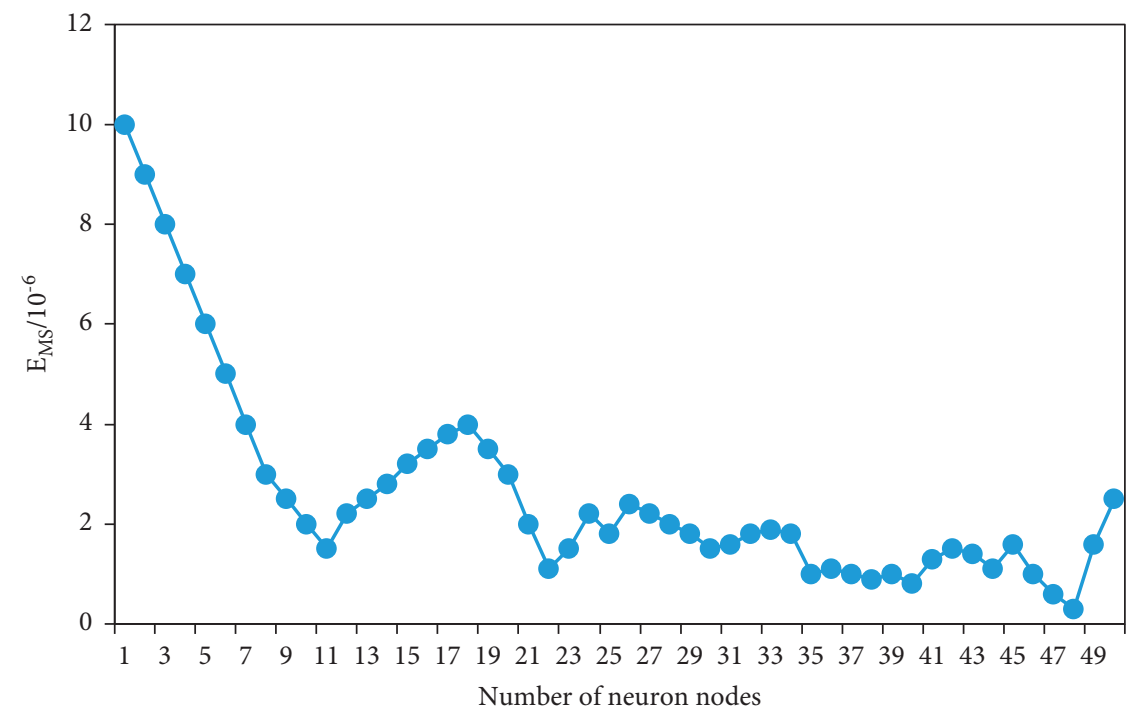

FIGURE 3: Network MSE distribution.

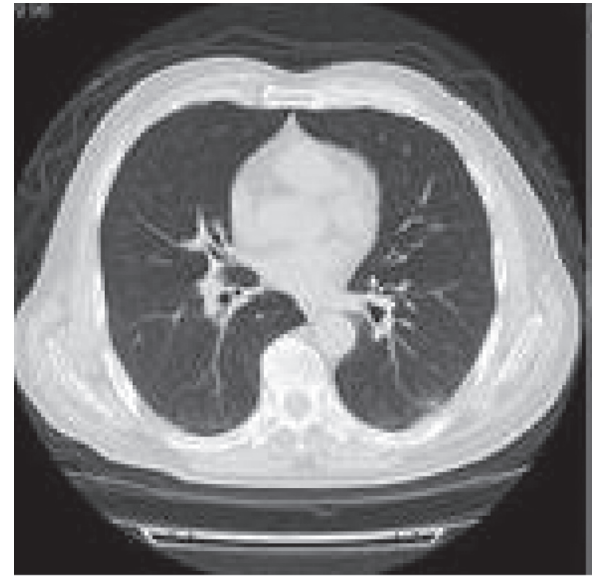

(a)

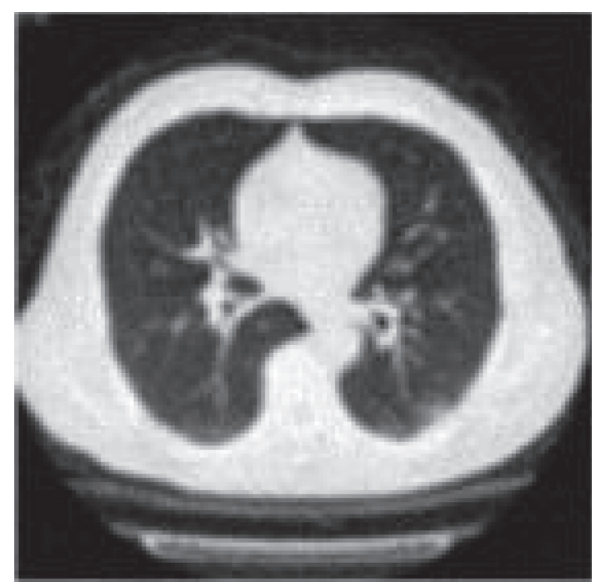

(c)

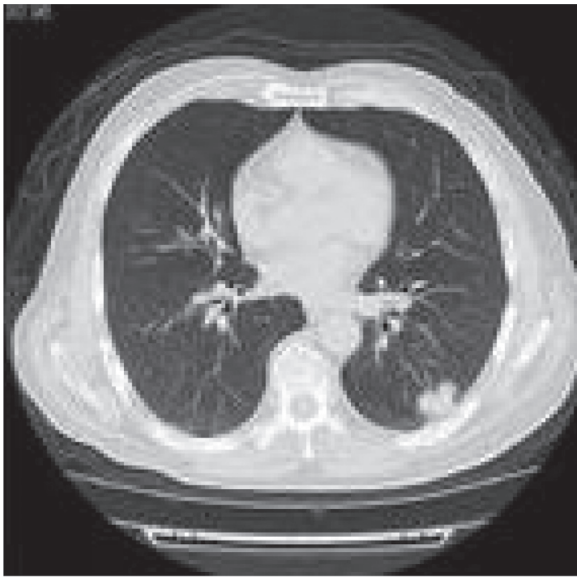

(b)

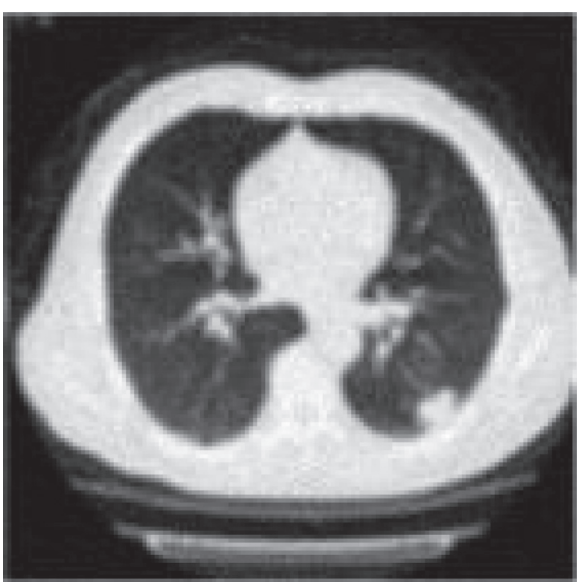

(d)

FIgURE 4: CT images of two patients with lung cancers.

lower compared to that in the control group $(P<0.05)$. After treatment, the effect of the observation group was much better than that of the control group.
3.5. Comparison of Curative Effect of Two Groups of Patients before and after Treatment. Obviously effective: patients had disappeared cough, shortness of breath, rales, and wheezing 


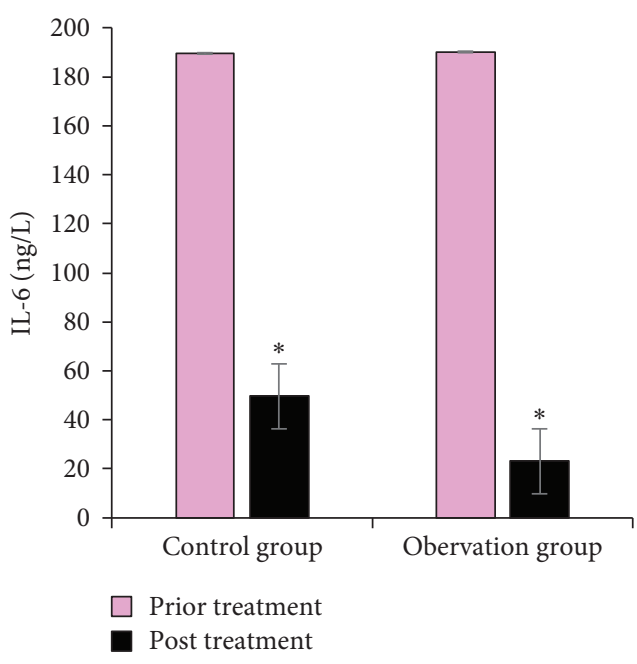

(a)

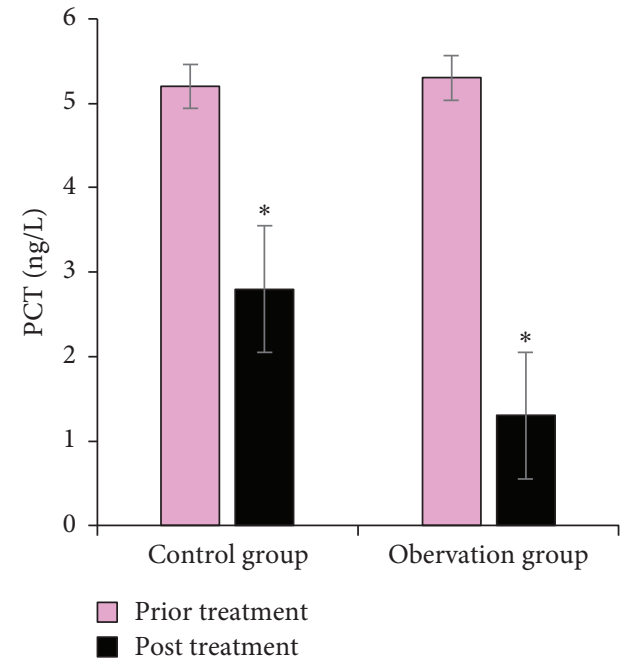

(c)

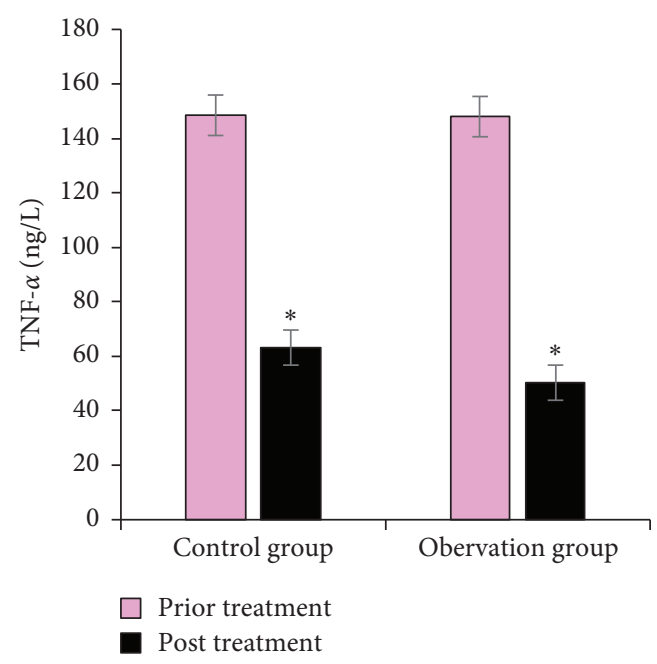

(b)

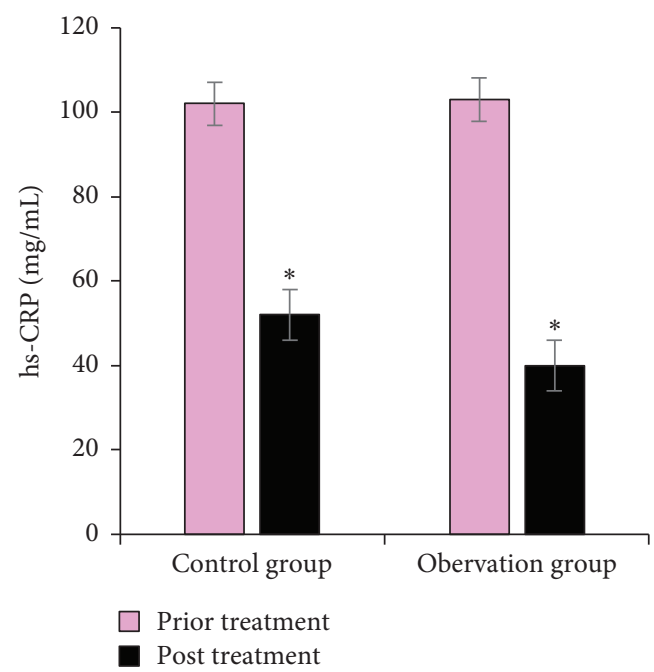

(d)

Figure 5: Comparison of inflammatory factor test results for patients in two groups. Note: ${ }^{*}$ indicates that the difference is statistically significant compared with that before treatment, $P<0.05$.

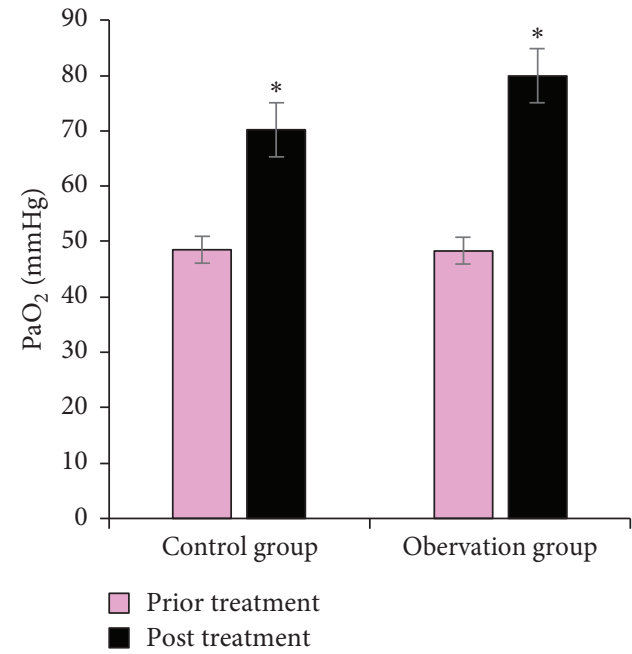

(a)

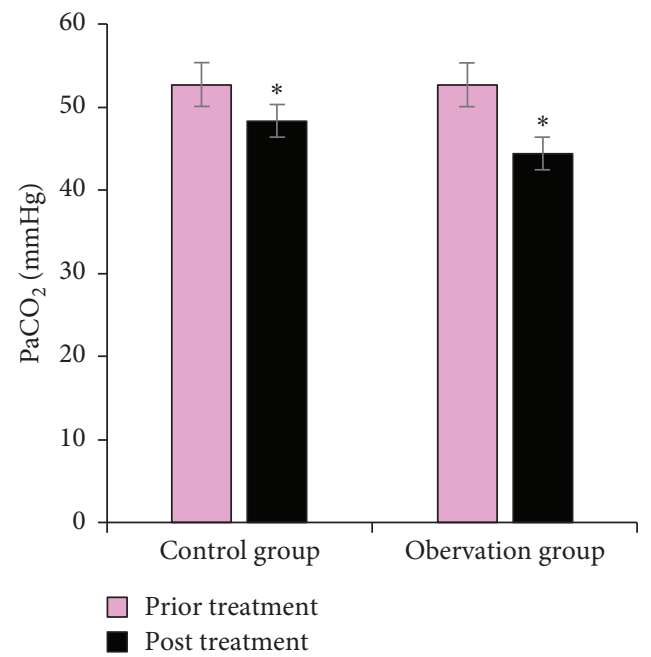

(b)

Figure 6: Comparison of $\mathrm{PaCO}_{2}$ and $\mathrm{PaO}_{2}$ before and after the treatment. Note: ${ }^{*}$ indicates that the difference is statistically significant compared with that before treatment, $P<0.05$. 
TABLE 2: Comparison of curative effect of two groups of patients ( $n \%)$.

\begin{tabular}{|c|c|c|c|c|c|}
\hline Group & Number & Obviously effective & Effective & Ineffective & Total effective rate \\
\hline Observation group & 40 & $23(57.5)$ & $16(40)$ & $1(2.5)$ & $39(97.5)^{*}$ \\
\hline Control group & 40 & $20(50)$ & $15(37.5)$ & $5(6.25)$ & $35(87.5)$ \\
\hline$X^{2}$ & & & & & 3.927 \\
\hline$P$ & & & & & 0.041 \\
\hline
\end{tabular}

Note. ${ }^{*}$ suggests that the difference is statistically obvious $(P<0.05)$.

and increased blood gas analysis, $\mathrm{PaO}_{2}$ rising, and $\mathrm{PaCO}_{2}$. Effective: the patient's clinical symptoms had been improved, and various indicators had been greatly improved. Ineffective: there was no improvement in clinical indicators or even worsening. The total effective rate was the sum of markedly effective and effective. Table 2 shows that the total effective rate of the observation group was $97.5 \%$, and the effective rate of the control group was $87.5 \%$. After treatment, the total effective rate of the observation group was greatly higher than that of the control group $(P<0.05)$.

3.6. Comparison of Quality of Life of Patients before and after Treatment. The FACT-L scores of the two groups before and after treatment were compared, and the results are shown in Figure 7. The scores of the two groups of patients had improved after treatment, and the scores of the observation group were much higher than those of the control group. After treatment, the FACT-L score was greatly higher than that before treatment $(P<0.05)$.

\section{Discussion}

The trend of population aging in China continues to deepen, and COPD has also become a threat to the health of the elderly [15]. Oxygen therapy is an important part of the clinical treatment of COPD patients. The traditional humidification bottle oxygen inhalation device has many shortcomings, such as poor humidification effect and low oxygen utilization, which will lead to complications such as nasal mucosal promotion and crusting, affecting the excretion of sputum. The HFNC treatment can make the inhaled oxygen enter the nasal cavity after heating, the gas flow that can pass through the device setting interface, and the human body temperature and humidity $[16,17]$. The study of Stéphan et al. [18] demonstrated that the great advantages, comfort, and compliance of the HFNC device are very good; it can reduce airway resistance and increase end-expiratory lung ventilation and positive pressure ventilation and other physiological characteristics. After all patients in this study were scanned by artificial intelligence $\mathrm{CT}$, patients in the observation group were treated with HFNC on the basis of anti-inflammatory. The results showed that the inflammatory factors (hs-CRP, PCT, TNF- $\alpha$, and IL6 ) of the patients were greatly reduced after treatment. The reduction effect of the observation group was better than that of the control group. This suggested that after receiving anti-inflammatory, phlegm, and other symptomatic treatments, HFNC treatment was more effective than simple anti-inflammatory and phlegm treatment. Inflammatory

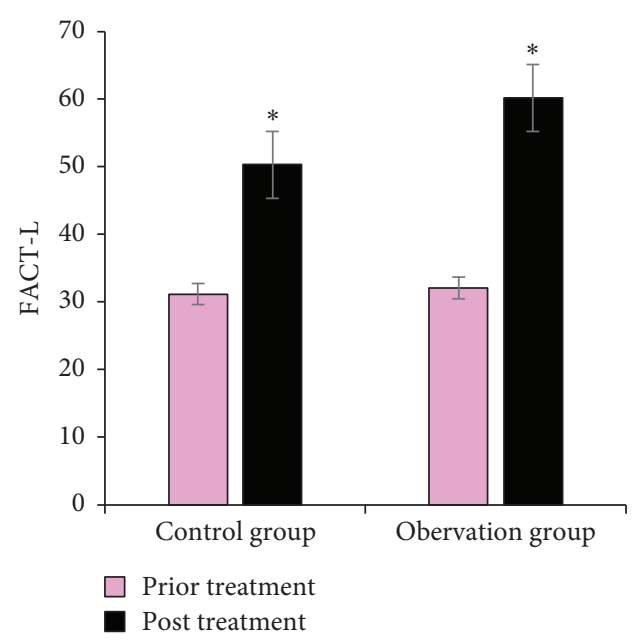

FIgURE 7: Comparison of FACT-L score of patients before and after the treatment. Note: ${ }^{*}$ indicates that the difference is statistically significant compared with that before treatment, $P<0.05$.

factors play a very important role in COPD complicated with lung cancer. Proinflammatory factors closely related to lung cancer include transforming growth factor $\beta$ (TGF- $\beta$ ), IL, TNF- $\alpha$, and cyclooxygenase 2 . In the course of COPD, IL- 6 and TNF- $\alpha$ can also directly or indirectly induce the proliferation and differentiation of airway epithelial cells, regulate the cell cycle, participate in the migration of inflammatory cells and intensify the oxidation reaction, stimulate the release of inflammatory factors, and trigger the progress of COPD.

PCT is a protein. If the body suffers from severe bacterial, fungal infection or multiple organ failure, PCT in the serum will increase observably. Studies have shown that serum PCT in patients with coinfection of malignant tumors is greatly increased. hs-CRP is a C-reactive protein in plasma. When the body is exposed to microbial invasion and other inflammatory stimuli, acute phase proteins will be stimulated, phagocyte function will be enhanced, and complement will be activated. The normal range of CRP is below $10 \mathrm{mg} / \mathrm{mL}$ [19]. The values in both groups of patients in this study were higher than this value. After treatment, the CRP content of the observation group was much lower than that of the control group. It indicated that the HFNC treatment can effectively reduce the content of PCT and CRP in the patient's body and inhibit inflammatory factors. Storgaard et al. [20] used HFNC treatment on COPD patients and found that this treatment reduced the exacerbation of lung diseases in COPD patients, and the patients' hospitalization symptoms were significantly reduced. The same conclusion 
was reached in this study. Before treatment, the $\mathrm{PaO}_{2}$ content of the control group and the observation group were $48.36 \mathrm{mmHg}$ and $48.52 \mathrm{mmHg}$. After treatment, the $\mathrm{PaO}_{2}$ content of the control group and the observation group was $70.18 \mathrm{mmHg}$ and $79.95 \mathrm{mmHg}$. After treatment, the $\mathrm{PaO}_{2}$ content of the observation group was dramatically higher in contrast to that of the control group $(P<0.05)$, and the $\mathrm{PaCO}_{2}$ content was visibly lower than that of the control group $(P<0.05)$. After treatment, the effect of the observation group was much better compared to that of the control group. This suggested that the addition of HFNC therapy on the basis of anti-inflammatory therapy can effectively improve the patient's blood gas status. In this study, the quality of life of the observation group before treatment was 32.2, the quality of life after treatment was 59.2, the quality of life of the control group was 31.6, and the quality of life after treatment was 49.2. The quality of life and total effective rate of the observation group were also significantly better than those of the control group. The total effective rate of the observation group was obviously better. For patients with advanced lung cancer or poor lung function, HFNC treatment can be added to the choice of anti-inflammatory, phlegm, and other symptomatic treatments to improve the quality of life of the patients.

\section{Conclusion}

In this study, artificial intelligence was applied to the CT treatment and diagnosis of patients with lung cancer and COPD, which effectively improved the efficiency of patient image feature extraction and classification. It was found in this study that, compared with the anti-inflammatory and phlegm treatment alone, the $\mathrm{PaO}_{2}$ and $\mathrm{PaCO}_{2}$ of patients had been greatly improved by HFNC therapy. It promoted the discharge of the sputum, improve respiratory function, and greatly reduce the inflammatory factors of the patients. The HFNC therapy can improve the patient's oxygen therapy comfort and quality of life, with a total effective rate of $97.5 \%$. After treatment, the quality of life in the observation group was increased by $45.69 \%$ compared with that before treatment, and the quality of life in the control group was increased by $35.77 \%$.

Although the evaluation model of the BPNN showed a certain reference value, there were still some shortcomings in practical applications, such as the inability of the test data to completely eliminate the interference of subjective factors, so the specific indicators can be standardized in the future. In addition, the sample size in this study was relatively small, and it is hoped to add more physiological and clinical data in the future to further expand the sample research support.

\section{Data Availability}

The data used to support the findings of this study are available from the corresponding author upon request.

\section{Conflicts of Interest}

The authors declare no conflicts of interest.

\section{Acknowledgments}

This work was supported by the Chenzhou City Joint Special Projects in the Hospital of Xiangnan College, Hunan Province (no. 2020XJ97).

\section{References}

[1] L. L. Carr, S. Jacobson, D. A. Lynch et al., "Features of COPD as predictors of lung cancer," Chest, vol. 153, no. 6, pp. 1326-1335, 2018.

[2] A. Nicolini, B. Grecchi, M. Ferrari-Bravo, and C. Barlascini, "Safety and effectiveness of the high-frequency chest wall oscillation vs intrapulmonary percussive ventilation in patients with severe COPD," International Journal of Chronic Obstructive Pulmonary Disease, vol. 13, pp. 617-625, 2018.

[3] L. Pisani, M. Astuto, I. Prediletto, and F. Longhini, "High flow through nasal cannula in exacerbated COPD patients: a systematic review," Pulmonology, vol. 25, no. 6, pp. 348-354, 2019.

[4] V. Pinto-Plata, C. Casanova, M. Divo et al., "Plasma metabolomics and clinical predictors of survival differences in COPD patients," Respiratory Research, vol. 20, no. 1, p. 219, 2019.

[5] J. Long, Y. Ouyang, H. Duan et al., "Multiple factor analysis of depression and/or anxiety in patients with acute exacerbation chronic obstructive pulmonary disease," International Journal of Chronic Obstructive Pulmonary Disease, vol. 15, pp. 1449$1464,2020$.

[6] H. Li, Z. Zeng, J. Cheng et al., "Prognostic role of NT-proBNP for in-hospital and 1-year mortality in patients with acute exacerbations of COPD," International Journal of Chronic Obstructive Pulmonary Disease, vol. 15, pp. 57-67, 2020.

[7] V. Oliveira Prados, T. Lima, L. Tavares da Silva et al., "Evaluation of the left ventricle in patients with COPD and nocturnal hypoxemia," Jornal Brasileiro de Pneumologia, vol. 46, no. 6, Article ID e20190136, 2020.

[8] M. E. Yuste, O. Moreno, S. Narbona, F. Acosta, L. Peñas, and M. Colmenero, "Efficacy and safety of high-flow nasal cannula oxygen therapy in moderate acute hypercapnic respiratory failure," Revista Brasileira de Terapia Intensiva, vol. 31, no. 2, pp. 156-163, 2019.

[9] T. Perez, G. Deslée, P. R. Burgel et al., "Predictors in routine practice of 6-min walking distance and oxygen desaturation in patients with COPD: impact of comorbidities," International Journal of Chronic Obstructive Pulmonary Disease, vol. 14, pp. 1399-1410, 2019.

[10] J. Bräunlich, D. Dellweg, A. Bastian et al., "Nasal high-flow versus noninvasive ventilation in patients with chronic hypercapnic COPD," International Journal of Chronic Obstructive Pulmonary Disease, vol. 14, pp. 1411-1421, 2019.

[11] J. Buekers, J. Theunis, P. De Boever et al., "Wearable finger pulse oximetry for continuous oxygen saturation measurements during daily home routines of patients with chronic obstructive pulmonary disease (COPD) over one week: observational study," JMIR mHealth and uHealth, vol. 7, no. 6, Article ID e12866, 2019.

[12] Y. Kim, J. M. Modrick, E. C. Pennington, and Y. Kim, "Commissioning of a 3D image-based treatment planning system for high-dose-rate brachytherapy of cervical cancer," Journal of Applied Clinical Medical Physics, vol. 17, no. 2, pp. 405-426, 2016.

[13] I. Ribeiro, H. Janssen, M. De Brabandere et al., "Long term experience with $3 \mathrm{D}$ image guided brachytherapy and clinical 
outcome in cervical cancer patients," Radiotherapy \& Oncology, vol. 120, no. 3, pp. 447-454, 2016.

[14] C. Guo, J. Lu, Z. Tian, W. Guo, and A. Darvishan, "Optimization of critical parameters of PEM fuel cell using TLBODE based on Elman neural network," Energy Conversion and Management, vol. 183, pp. 149-158, 2019.

[15] J. Bräunlich, F. Mauersberger, and H. Wirtz, "Effectiveness of nasal highflow in hypercapnic COPD patients is flow and leakage dependent," BMC Pulmonary Medicine, vol. 18, no. 1, p. 14,2018 .

[16] S. McKinstry, J. Singer, J. P. Baarsma, M. Weatherall, R. Beasley, and J. Fingleton, "Nasal high-flow therapy compared with non-invasive ventilation in COPD patients with chronic respiratory failure: a randomized controlled crossover trial," Respirology, vol. 24, no. 11, pp. 1081-1087, 2019.

[17] N. Kirici Berber, Ö. Yetkin, T. Kilic, İ. Berber, and M. Özgel, "The effects of home oxygen therapy on energy metabolism in patients with COPD," International Journal of Chronic Obstructive Pulmonary Disease, vol. 13, pp. 1577-1582, 2018.

[18] F. Stéphan, B. Barrucand, P. Petit et al., "High-flow nasal oxygen vs noninvasive positive airway pressure in hypoxemic patients after cardiothoracic surgery," Journal of the American Medical Association, vol. 313, no. 23, pp. 2331-2339, 2015.

[19] D. M. Magdy and A. Metwally, "Effect of average volumeassured pressure support treatment on health-related quality of life in COPD patients with chronic hypercapnic respiratory failure: a randomized trial," Respiratory Research, vol. 21, no. 1, p. $64,2020$.

[20] L. H. Storgaard, H. Hockey, B. S. Laursen, and U. M. Weinreich, "Long-term effects of oxygen-enriched high-flow nasal cannula treatment in COPD patients with chronic hypoxemic respiratory failure," International Journal of Chronic Obstructive Pulmonary Disease, vol. 13, pp. 11951205, 2018. 